\title{
Rupture of thin liquid films under the influence of external heat flux
}

\author{
K. V. Meredith \& J. de Vries \\ FM Global, Research Division, USA
}

\begin{abstract}
A thin liquid film completely covering a solid surface has the potential to rupture into rivulets when exposed to external heat flux due to thermocapillary instabilities and vaporization. This has implications for many industrial applications, but particularly for fire suppression. Film rupture drastically reduces the wetted surface area thereby exposing the solid surface to large incident heat fluxes. Recently, a computational fluid dynamics (CFD) model-FireFOAM-has been developed for simulating fire suppression phenomena. The purpose of this study is to investigate and validate FireFOAM's thin film model for predictions of critical heat flux required to induce film rupture. To that extent, vertically flowing film experiments with flow rates ranging form $8.7 \mathrm{~g} / \mathrm{m} / \mathrm{s}$ to $41.3 \mathrm{~g} / \mathrm{m} / \mathrm{s}$ and external heat flux extending from $5 \mathrm{~kW} / \mathrm{m}^{2}$ to $45 \mathrm{~kW} / \mathrm{m}^{2}$ have been simulated. The film heats up non-uniformly and eventually ruptures due to thermocapillary instabilities. Dry regions are formed on the panel as the film pulls together as rivulets. For a given flow rate, the radiative heat flux was varied to identify the 'critical heat flux'. Good qualitative and quantitative match was achieved between model and experiments. At the lower flow rates, even a slight incident heat flux would cause the film to break into rivulets. As the flow rate increased, stronger and stronger heat flux values were necessary to cause film rupture. At very high flow rates, the film remained continuous over the range of heat flux tested. Film rupture was shown to be sensitive to the film inlet conditions.

Keywords: fire suppression, computational fluid dynamics, thin-film assumption, partial wetting, contact line, critical heat flux, conjugate heat transfer.
\end{abstract}




\section{Introduction}

The fire suppression community has long been interested in identifying the controlling parameters for preventing the propagation of fire through combustible materials [1]. In most common industrial applications, fire sprinklers have been employed for controlling the spread of fire [2]. Fire sprinklers activate in the presence of a fire and subsequently apply water to the fire and surrounding surfaces (a process known as prewetting). Combustible solids, when covered by a thin layer of water, are extremely difficult to ignite. Thus, as long as the materials surrounding a fire can be maintained in a sufficiently wet state, the fire will be contained to the original volume.

Prewetting assists in preventing the spread of fire [3] by the following two methods. First, the liquid water film blocks most thermal radiation emanating from the fire plume that is incident to the un-ignited surface; liquid water is fairly opaque to thermal radiation in the wavelengths seen in typical fires [4]. Secondly, the water film convectively cools the solid, keeping it below the solid pyrolysis temperatures. Thus, maintaining a continuous thin liquid film over the solid, combustible surfaces surrounding a fire is paramount to minimizing fire spread.

As is typical of thin liquid films flowing over vertical surfaces, two main flow regimes exist. First, for high flow rates the film will completely cover the wetted surface [5]. This is known as the completely flooded regime. For low flow rates, the film will flow in the form of individual rivulets on the surface [6]. This is known as the partially wetted flow regime. The area between the rivulets will be dry, and thus directly exposing the solid surface to external thermal radiation heat transfer [7]. Obviously, the dry area between rivulets will not experience convective cooling from the film.

Considering a completely flooded surface, surface-tangential temperature gradients, due to non-uniformity of film thickness and incident heat flux, will exist on surface of a thin film in fire suppression environments. These lateral temperature gradients result in surface tension induced thermocapillary instabilities [8], leading to local thinning of the film and ultimately resulting in film rupture. Thermocapillary instabilities, coupled with vaporization of the film, result in a reduced wetted area fraction for the solid surface. The ability to understand and predict this phenomenon is important for modeling the effectiveness of water suppression.

Recently, a computational fluid dynamics (CFD) model, FireFOAM, has been developed and validated for simulating fire suppression. The CFD model is based on the open source framework, OpenFOAM ${ }^{\circledR}$ [9]. Models for large-eddy simulation (LES) gas phase combustion [10], thermal radiation heat transfer from soot emission [11], and solid-phase pyrolysis [12] are included to simulate fire growth. In order to simulate fire suppression, the multiphase flow aspects of fire suppression need to be simulated as well. To this extent, a Lagrangian transport model simulating water droplets from the fire sprinkler through the gas-phase fire 
plume to the burning and/or unburnt surfaces is included. The water droplets, upon arriving at solid surfaces, are adsorbed onto the surface in the form of a thin film.

The thin-film model, described in detail elsewhere [13-16], consists of equations for mass, momentum, and energy conservation. These equations are solved on the solid surfaces to track the transport of water and to account for the interfacial interaction of the thin liquid film with the solid combustible surfaces and the gas-phase fire. The film model has been validated for many separate phenomena. Validation has been performed for partially wetted flow over vertical surfaces in the absence of external heat flux [13]. Interaction of impinging droplets on the film and subsequent splashing was also validated [14]. Validation of the film model in the flow of water over an array of boxes stacked in a rack-storage arrangement also occurred [15]. Finally, conjugate heat transfer and vaporization predictions of the liquid film model were shown to match experimental data very well [16]. The motivation for this study is to validate the thin film model's ability to capture thermocapillary instabilities and subsequent film rupture.

\section{Numerical model}

\subsection{Thin film model}

The thin film model has been described previously in much detail $[13,15]$. Here, only the relevant portions of the model necessary for understanding this validation study are presented. Essentially, the thin film model consists of transport equations for mass continuity, momentum conservation, and energy conservation. These three equations are, respectively,

$$
\begin{gathered}
\frac{\partial \rho \delta}{\partial t}+\nabla_{s} \cdot[\rho \delta \mathbf{U}]=S_{\rho \delta} \\
\frac{\partial \rho \delta \mathbf{U}}{\partial t}+\nabla_{s} \cdot[\rho \delta \mathbf{U U}]=\underbrace{-\delta \nabla_{s} p}_{\text {pressure based }}+\underbrace{\mathbf{S}_{\rho \delta \mathbf{U}}}_{\text {stress based }} \\
\frac{\partial \rho \delta h}{\partial t}+\nabla_{s} \cdot[\rho \delta \mathbf{U} h]=S_{\rho \delta h}
\end{gathered}
$$

In these transport equations, $\rho$ is liquid density, $\delta$ is film thickness, $\mathbf{U}$ is film velocity, and $\nabla_{s}$ is the vector differential operator tangential to the surface, $\left(\frac{\partial}{\partial x}, \frac{\partial}{\partial y}\right)^{\top}$. The right hand sides of Equations 1-3 represent the respective source terms. In this validation study, the source term of the mass continuity equation, $S_{\rho \delta}$, consists only of the vaporization rate per unit surface area.

For the purposes of this study, the pressure based momentum terms, $-\delta \nabla_{s} p$, are not relevant, but have been described elsewhere [13]. The stress term, $\mathbf{S}_{\rho \delta \mathbf{U}}$, includes the viscous surface shear stresses $\left(\boldsymbol{\tau}_{g}, \boldsymbol{\tau}_{w}\right)$, gravity body force $\left(\rho \mathbf{g}_{t} \delta\right)$, and stress related to a contact-angle force $\left(\boldsymbol{\tau}_{\theta}\right)$.

$$
\mathbf{S}_{\rho \delta \mathbf{U}}=\boldsymbol{\tau}_{g}+\boldsymbol{\tau}_{w}+\rho \mathbf{g}_{t} \delta+\boldsymbol{\tau}_{\theta}+\boldsymbol{\tau}_{\text {mar }}
$$


The submodels for $\boldsymbol{\tau}_{g}, \boldsymbol{\tau}_{w}$, and $\rho \mathbf{g}_{t} \delta$ have been described elsewhere [15]. The submodel for $\boldsymbol{\tau}_{\theta}$, in which partially wetted treatment occurs, has also been previously described [13], but a brief description will be given here for completion. The contact angle shear stress term is defined as

$$
\boldsymbol{\tau}_{\theta}=\frac{\sigma\left(1-\cos \theta_{E}\right)}{\Delta_{c l}}
$$

where $\Delta_{c l}$ is a characteristic width for applying the contact angle shear stress, and $\theta_{E}$ is the contact angle.

The thermocapillary shear stress, $\boldsymbol{\tau}_{\text {mar }}$, is defined as

$$
\boldsymbol{\tau}_{\text {mar }}=-\nabla_{s} \sigma\left(T_{f}\right)
$$

where $\sigma$ represents surface tension as a function of film temperature, $T_{f}$. The vaporization model has also been previously described elsewhere [15].

\subsection{Solid heat conduction model}

In order to accurately simulate the film thermal back-boundary condition, a conjugate heat transfer model was introduced into the film model to account for the heat conduction within the panel. The numerical model for the solid consists of a straightforward three-dimensional heat conduction model. Solid thermal properties of heat capacity, thermal conductivity, and density were provided as inputs to the numerical model. The heat transfer in the solid was coupled to the film-model and to the gas-phase model.

\section{Experimental setup}

As a means of understanding the response of film flow under non-adiabatic conditions, a radiant panel experiment was performed by de Vries et al. [7] The goal of this experiment was to study film rupture and measure the vaporization rate as a function of radiant heat flux. The vertical panel available for film flow was $51 \mathrm{~cm}$ wide by $122 \mathrm{~cm}$ long and made from $1.2 \mathrm{~mm}$ thick aluminum. The back side of the aluminum was insulated with $5 \mathrm{~cm}$ Kaowool ceramic fiber. Specifically, the submodels for heat transfer, vaporization, and thermocapillary effects were able to be tested in this configuration independent of the pyrolysis and charring that would have been experienced with a combustible solid used as the panel material.

The front surface was sprayed with carbon black paint, giving the surface a radiative absorptivity of 0.96 . A shield with a rectangular opening $(46 \mathrm{~cm}$ wide by $41 \mathrm{~cm}$ high) was placed several centimeters in front of the panel surface in order to provide a controlled area of incident radiative heat flux. The heat flux to the panel was varied by moving a radiant heater in the direction normal to the panel. Tested film flow rates ranged from $\Gamma=7.0 \mathrm{~g} / \mathrm{m} / \mathrm{s}$ to $\Gamma=41.1 \mathrm{~g} / \mathrm{m} / \mathrm{s}$, where the values have been normalized by the width of the panel. Radiant heat flux values ranged varied between $\dot{q}_{i n c}^{\prime \prime}=20 \mathrm{~kW} / \mathrm{m}^{2}$ to $\dot{q}_{i n c}^{\prime \prime}=40 \mathrm{~kW} / \mathrm{m}^{2}$. Heat flux levels were 
validated through a separate set of calibration experiments using Schmidt-Boelter type heat flux gauges. An initial, flooded film flow condition was established at each flow rate prior to applying the heat source.

\section{Validation specific boundary conditions}

\subsection{Convective heat transfer}

Convective heat flux from film to wall, $\dot{q}_{f w}^{\prime \prime}$, is given by an expression for heat conduction from the film to the wall:

$$
\dot{q}_{f w}^{\prime \prime}=\frac{2 \lambda_{f}}{\delta}\left(T_{f}-T_{w}\right)
$$

where $\lambda_{f}$ represents the thermal conductivity of water, and $\delta$ is the film thickness, $T_{f}$ is the temperature of the film, and $T_{w}$ represents the wall surface temperature. Convective heat transfer from the gas to film, $\dot{q}_{g f}^{\prime \prime}$, is represented as

$$
\dot{q}_{g f}^{\prime \prime}=h_{g f}\left(T_{f}-T_{g}\right)
$$

Convective heat transfer from the gas phase to the solid conduction model was accomplished through the similar use of a convective coefficient, $h_{g w}$. The value of $h_{g f}$ used in the simulations was $40 \mathrm{~kW} / \mathrm{m}^{2}$.

The back side of the panel assumes an adiabatic boundary condition, due to the insulating Kaowool layer. Due to the thinness and high thermal conductivity of the panel, heat transfer occurred mainly in the surface tangential direction.

\subsection{Radiative heat transfer}

The experimental setup, as described earlier, warrants prescription of a constant incident radiative heat flux on the front side of the panel. The source term for thermal radiation absorption at the interface of the film and the gas phase is as follows:

$$
S_{\rho \delta h, r a d}=\alpha_{f} \dot{q}_{i n c}^{\prime \prime}
$$

where $\alpha_{f}$ represents the film thermal absorptivity and $\dot{q}_{i n c}^{\prime \prime}$ represents the incident radiative heat flux. Re-radiation is neglected, as the film temperature remains relatively low (from a thermal radiation perspective) due to being bounded by the boiling point of water at the upper limit.

At the interface of the gas phase and the panel, the radiation boundary condition is treated as

$$
S_{\rho \delta h, r a d}=\alpha_{s} \dot{q}_{i n c}^{\prime \prime}-\varepsilon_{s} \sigma T_{w}^{4}
$$

where $\alpha_{s}$ represents the thermal absorptivity of the solid, $\varepsilon_{s}$ represents the thermal emissivity of the solid, $\sigma$ represents the Stefan-Boltzmann constant, and $T_{w}$ represents the solid panel surface temperature. 


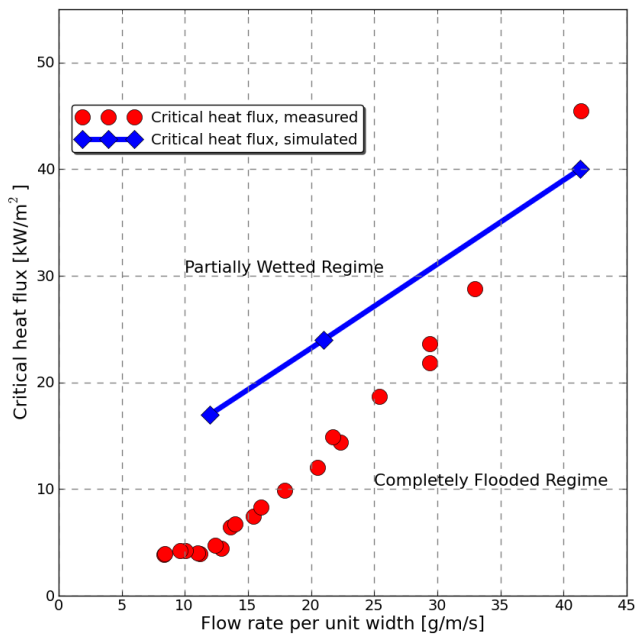

Figure 1: Critical heat flux predictions compared to measured values.

\subsection{Results}

The radiant panel tests were initialized with a film that was continuous over the entire panel surface. As long as the heat flux remained relatively low, the film would remain continuous. However, experimental observation showed that the film would rupture into rivulet flow when exposed to a high enough heat flux. The heat flux at which this transition from continuous film flow to rivulet flow is termed here as the 'critical heat flux'. For the range of conditions the experiment covered, a map of the critical heat flux, shown in Figure 1, was created as a function of film flow rate. The region lying above the curve represents the partially wetted regime, where rivulet flow exists. The region lying below the curve represents the completely flooded regime, where the entire surface is covered with the thin film.

To ascertain the critical heat flux predictions of the model, a case was simulated for each mean inlet flow rate. The simulations were designed to mimic the experiment. First, a steady flow was established at the given flow rate. Next, the external radiation was applied at a value well below the critical heat flux value. The heat flux was then slowly increased (at each heat flux level allowing the film to reach a pseudo steady-state condition) until the point at which the film rupture occurred. These results are shown in Figure 1 along with the experimental measurements.

The simulated predictions for critical heat flux were found to depend upon the specified inlet condition for the film model. In order to facilitate the film rupture in the simulations, a non-uniform film inlet condition was required. Non-uniformity in the film flow and irradiation in the experimental setup is believed to contribute to 
film rupture due to thermocapillary instabilities. To this end, a sinusoidal variation of film thickness (and thereby velocity), $\delta$, was specified along the inlet at the top of the panel. An example of such a variation is

$$
\delta(x)=\delta_{\text {mean }}+a \sin (\omega 2 \pi x)
$$

where the value of $\delta_{\text {mean }}$ determines the overall mass flow rate at the inlet, while $\omega$ was chosen as $20 \mathrm{~m}^{-1}$, $a$ was chosen as $0.02 \mathrm{~mm}$, and $x$ represents the location across of the inlet. For reference, a typical inlet mean film thickness for a $40 \mathrm{~g} / \mathrm{m} / \mathrm{s}$ flow rate is approximately $0.21 \mathrm{~mm}$. The introduction of this type of functionality at the inlet produced the simulated critical heat flux curve shown in Figure 1. At lower flow rates, the critical heat flux is lower. At higher and higher flow rates, the critical heat flux increases. As $a$ is increased, the simulated critical heat flux decreases. The simulated critical heat flux behaves linearly with increasing flow rate, while experimental observation shows a more 'parabolic' trend.

Presently, there is little experimental basis for this inlet condition, other than the fact that non-uniformities must exist in the flow and/or imposed heat flux in order to create temperature gradients tangentially along the surface. Further experimental characterization of the film conditions in the radiant panel would provide a clearer experimental basis for this assumption.

\subsection{Temperature and film thickness}

Figure 2 shows sample film thickness and film temperature results for the radiant panel under ruptured and un-ruptured film scenarios. The rectangular window represents the area of the panel exposed to external irradiation. The film temperature rises upon entering this region of the panel. Due to non-uniformities in the film thickness (and therefore film velocity) across the width of the panel, the film heats up non-uniformly and eventually ruptures due to thermocapillary instabilities. Dry regions are formed on the panel (shown in blue on the film thickness contours) as the film pulls together as rivulets. The temperature of the film is constrained to the boiling point of water $(\sim 373.15 \mathrm{~K})$ at ambient pressure. The aluminum temperature in the dry regions rises to values as high as $500 \mathrm{~K}$ at the peak, depending on heat flux. Essentially, much of the radiative energy absorbed by the solid panel is conducted laterally through the aluminum and finally into the film. The temperature of the panel is kept low in the vicinity of the rivulets. The dry regions of the panel show temperatures far in excess of the boiling point of water. Relatively high values of film-to-wall heat transfer maintain the film and wall temperatures in near equilibrium, separated at most by $1-2 \mathrm{~K}$.

Thermocapillary stresses tend to cause rivulets to narrow in regions of heat transfer from the panel to the rivulet. Contrarily, in regions of heat transfer from the rivulet to the panel, the rivulets are caused to widen. This effect can be observed in Figures $2 \mathrm{a}, 2 \mathrm{~b}$, and $2 \mathrm{~d}$. The reason for this is related to the temperature gradient laterally across the rivulet. In the first instance, where the rivulet is being heated on the edges by the panel, the high surface tension near the center of the rivulet pulls the lower surface tension fluid near the rivulet edge towards the center. On 

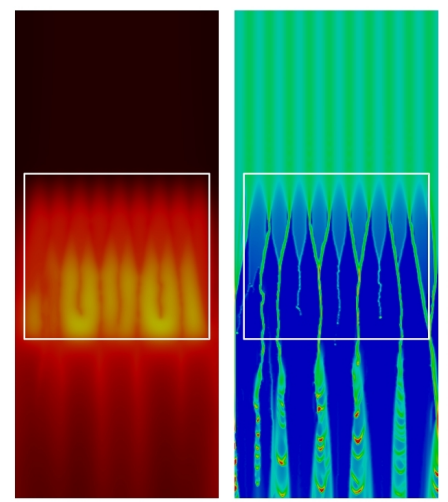

(a) $8.7 \mathrm{~g} / \mathrm{m} / \mathrm{s}, 20 \mathrm{~kW} / \mathrm{m}^{2}$
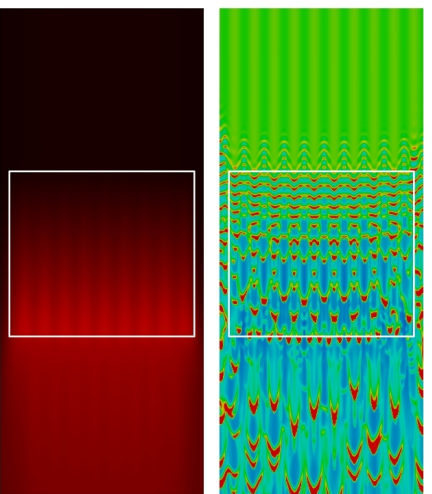

(c) $41.3 \mathrm{~g} / \mathrm{m} / \mathrm{s}, 20 \mathrm{~kW} / \mathrm{m}^{2}$
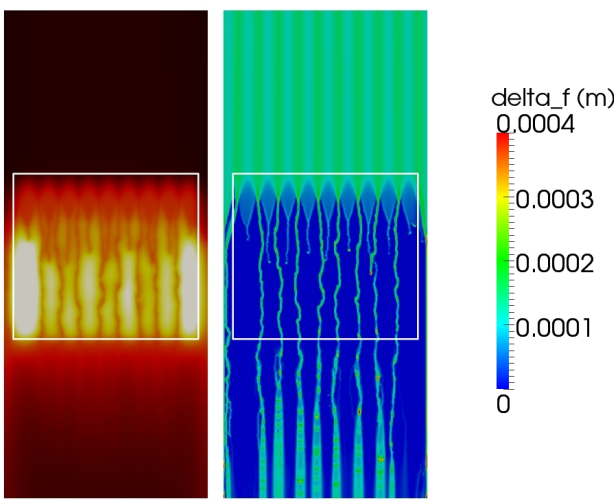

(b) $8.7 \mathrm{~g} / \mathrm{m} / \mathrm{s}, 40 \mathrm{~kW} / \mathrm{m}^{2}$
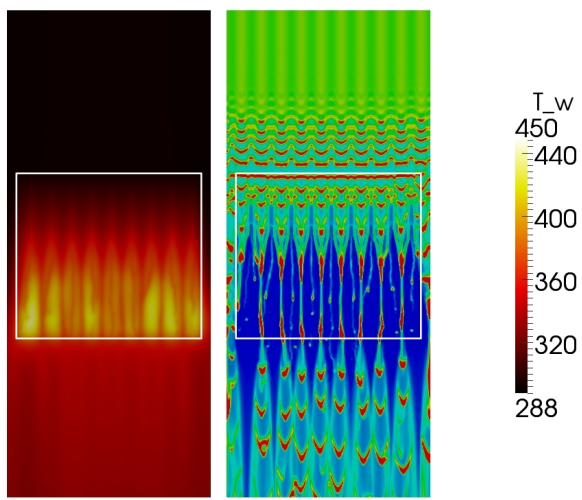

(d) $41.3 \mathrm{~g} / \mathrm{m} / \mathrm{s}, 40 \mathrm{~kW} / \mathrm{m}^{2}$

Figure 2: Panel temperature (left) and film thickness (right) predictions under four combinations of heat flux and flow rate. The area exposed to $\dot{q}_{i n c}^{\prime \prime}$ is shown in the rectangular overlay.

the other hand, when the rivulet is at an elevated temperature in relation to the panel, the edges of the rivulet are cooled by the panel and tend to cause the rivulet to widen due to the gradient in surface tension.

\subsection{Thermocapillary instabilities}

Figure 3 shows temperature and film thickness for the early stages of film thinning on a portion of the panel near the top of the irradiated rectangular region. In addition, vectors showing the direction of the thermocapillary stress term are shown. These thermocapillary stress vectors always point in the direction of increasing temperature. This figure illustrates a few of the key concepts important for understanding film rupture due to thermocapillary instabilities. 
Non-uniformities in the flow and incident heat flux create temperature gradients laterally across the film. The surface tension of water, being inversely proportional to temperature, yields thermocapillary stresses (cf. Equation 6), and result in subsequent movement of the film from hot regions to cold regions. As the film thins, the temperature continues to rise due to the longer residence time of the fluid (i.e. thinner film equals lower velocity). Vaporization is a strong function of temperature, and as the film thins it vaporizes more rapidly. Eventually the film becomes thin enough that it ruptures and a dry patch is formed. Thus, film rupture due to thermocapillary instabilities occurs from the combined interaction of heat, mass, and momentum transfer. In other words, thermocapillary induced film rupture not only depends on the mean values of film flow rate and incident heat flux, but also depends highly on non-uniformities within the film flow and also in the incident heat flux.
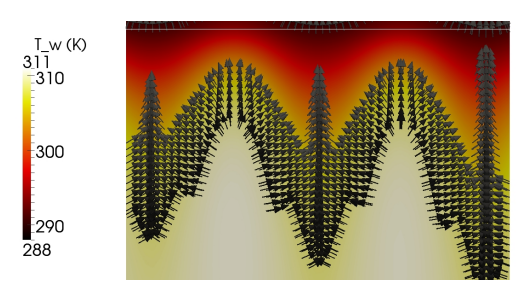

(a)
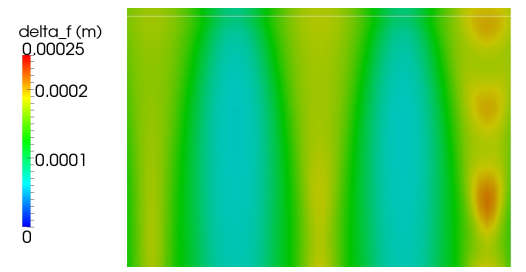

(b)

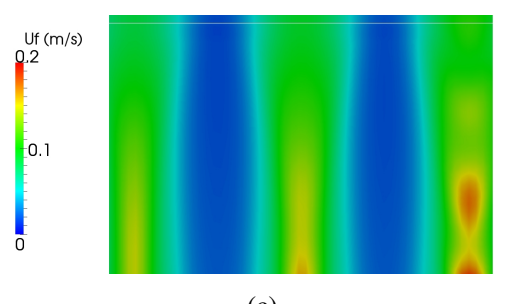

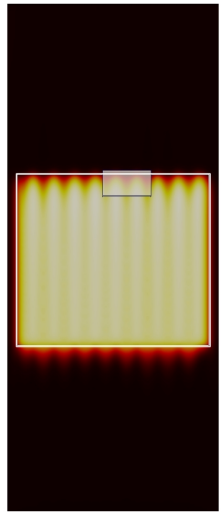

(d)

Figure 3: Details of early stages of film thinning: (a) thermocapillary stress vectors and film temperature contours, (b) film thickness contours, and (c) film velocity contours for a region near the top of the rectangular section, highlighted in (d). Flow rate of $8.7 \mathrm{~g} / \mathrm{m} / \mathrm{s}$ and heat flux of $40 \mathrm{~kW} / \mathrm{m}^{2}$. 


\subsection{Evaporation}

Inclusion of the conjugate heat transfer to the aluminum panel was important in obtaining good agreement with vaporization rate measurements. Without accounting for the lateral heat conduction in the panel from the dry regions to the wetted regions, the vaporization rate was grossly under predicted for partially wetted flow. The vaporization rate was measured as a function of water flow rate and incident heat flux. A comparison between the film model predictions and the measured values for the lowest and highest applied flow rates is shown in Figure 4.

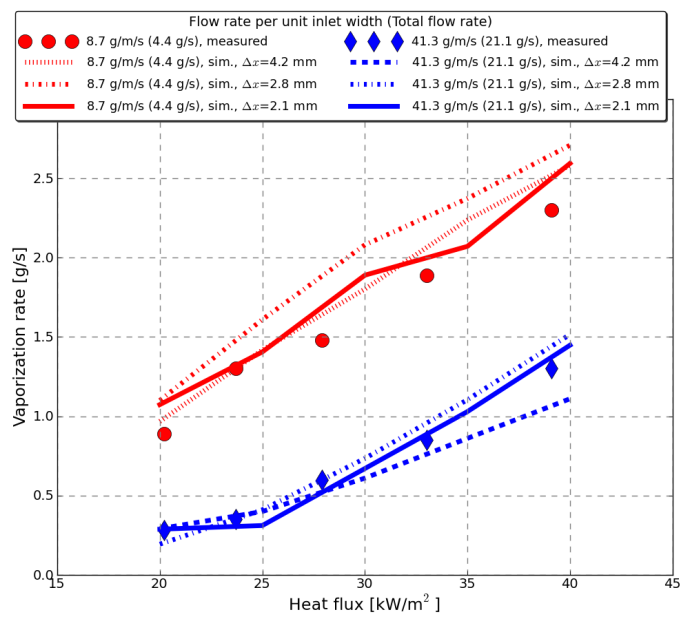

Figure 4: Validation of vaporization model against radiant panel measurements.

The grid sensitivity is also shown in this Figure 4 showing vaporization rates computed for each flow rate at increasing levels of grid refinement. The vaporization rate was found to be fairly independent of grid refinement. The vaporization rate is slightly over predicted at high heat flux and low flow rate. For high heat flux and high flow rate, the vaporization is slightly under predicted. Still, the overall trend in the predicted vaporization rate matches the experimental behavior quite well.

\section{Conclusions}

This study provided insight into the behavior of thin liquid flowing films under the influence of an external irradiative heat flux. Thin liquid films completely covering a solid surface were shown to have the potential to rupture into rivulets when exposed to external heat flux due to thermocapillary instabilities and vaporization. 
The implications for fire suppression are that film rupture drastically reduces the wetted surface area thereby exposing the solid surface to large incident heat fluxes. FireFOAM, a recently developed CFD code for simulating fire suppression, was used in this study to validate the thin film model predictions for critical heat flux, vaporization, and conjugate heat transfer required to induce film rupture. The film rupture experiments of de Vries et al. [7] were simulated. The vertically flowing film heated up non-uniformly and eventually ruptured due to thermocapillary instabilities. Dry regions were formed on the panel as the film pulls together as rivulets. For each flow rate, the radiative heat flux was varied in order to identify the predicted 'critical heat flux'. Good qualitative and quantitative match was achieved between model and experiments. At the lower flow rates, even a slight incident heat flux would cause the film to break into rivulets. As the flow rate increased, stronger and stronger heat flux values were necessary to cause film rupture. At very high flow rates, the film remained continuous over the range of heat flux tested. Film rupture was shown to be sensitive to the film inlet conditions.

\section{Acknowledgements}

This work was funded by FM Global's Strategic Research Program for Fire and Suppression Modeling. Andy Heather is acknowledged for the development of the underlying film model framework. OpenFOAM is a registered trademark of the OpenFOAM Foundation.

\section{References}

[1] Corlett, R. \& Williams, F., Modeling direct suppression of open fires. Fire Safety Journal, 1(6), pp. 323 - 337, 1979.

[2] Yates, C., Fire sprinkler systems. Plant Engineer's Ref. Book (Second Edition), ed. D.A. Snow, Butterworth-Heinemann: Oxford, 2003.

[3] Nam, S., Actuation of sprinklers at high ceiling clearance facilities. Fire Safety Journal, 39(7), pp. 619 - 642, 2004.

[4] Kutz, M., (ed.) Mechanical Engineers' Handbook (2nd Edition). John Wiley $\&$ Sons, 1998. Contains alpha $=0.96$ for water.

[5] Nusselt, W., Die oberflachenkondensation des wasserdampfes. Zeitschrift des Vereines Deutscher Ingenieure, 60, pp. 541-546 and 569-575, 1916.

[6] Mikielewicz, J. \& Moszynskl, J., Minimum thickness of a liquid film flowing vertically down a solid surface. International Journal of Heat and Mass Transfer, 19(7), pp. 771 - 776, 1976.

[7] de Vries, J., Xin, Y. \& Meredith, K.V., An experimental study of fire suppression physics for sprinkler protection. Fire Safety Science, 10, pp. 429 442, 2011.

[8] Slade, D., Veremieiev, S., Lee, Y. \& Gaskell, P., Gravity-driven thin film flow: The influence of topography and surface tension gradient on rivulet formation. Chemical Engineering and Processing, 2012. 
[9] http://www.openfoam.org/, April, 2013.

[10] Wang, Y., Chatterjee, P. \& de Ris, J.L., Large eddy sim. of fire plumes. Proceedings of the Combustion Institute, 33(2), pp. 2473 - 2480, 2011.

[11] Chatterjee, P., de Ris, J.L., Wang, Y. \& Dorofeev, S.B., A model for soot radiation in buoyant diffusion flames. Proceedings of the Combustion Institute, 33(2), pp. 2665-2671, 2011.

[12] Chaos, M., Khan, M.M. \& Dorofeev, S.B., Pyrolysis of corrugated cardboard in inert and oxidative environments. Proceedings of the Combustion Institute, 34(2), pp. 2583 - 2590, 2012.

[13] Meredith, K.V., Heather, A., de Vries, J. \& Xin, Y., A numerical model for partially-wetted flow of thin liquid films. Computational Methods in Multiphase Flow, VI, pp. 239-250, 2011.

[14] Meredith, K., Zhou, X. \& Heather, A., A spray interaction model with application to surface film wetting. 12th International Conference on Liquid Atomization and Spray Systems, 2012.

[15] Meredith, K.V., Xin, Y. \& de Vries, J., A numerical model for simulation of thin-film water transport over solid fuel surfaces. Fire Safety Science, 10, pp. 415-428, 2011.

[16] Meredith, K.V., de Vries, J., Wang, Y. \& Xin, Y., A comprehensive model for simulating the interaction of water with solid surfaces in fire suppression environments. Proceedings of the Combustion Institute, 34(2), pp. 27192726, 2013. 